\title{
Biologie des pucerons des céréales dans l'ouest de la France. V. - Influence de la date de semis du blé d'hiver sur les modalités d'infestation des parcelles par Rhopalo- siphum padi (L.), Sitobion avenae (F.) et Metopolo- phium dirhodum (Wlk.) et sur le développement de leurs populations au printemps
}

Charles-Antoine DEDRYVER \& Sylvie TANGUY

I.N.R.A., Laboratoire de Zoologie, Centre de Recherches de Rennes, B.P. 29, F 35650 Le Rheu

En 1977, 1978, 1979, 1980 et 1982, on a comparé le développement des populations de Sitobion avenae (F.), Metopolophium dirhodum (Wlk.) et Rhopalosiphum padi (L.) au cours de l'automne et du printemps suivant, sur des parcelles de blé d'hiver semées précocement (octobre) et tardivement (novembre). Les semis précoces sont, dans tous les cas, contaminés avant l'hiver par $R$. padi et $S$. avenae. Les semis tardifs ne sont jamais contaminés par les pucerons avant le début du printemps suivant. Lorsque l'hiver est particulièrement rigoureux, comme cela a été le cas en 1978-1979, les virginipares hivernant sur les semis précoces sont détruits et les 2 semis sont recontaminés au même moment au printemps. Les pucerons y développent alors des populations de tailles très semblables, voire nettement plus fortes sur les semis tardifs (cas de 1979). Dans tous les autres cas $(1978,1980,1982)$, des populations de virginipares des 3 espèces passent l'hiver sur les semis précoces et se développent très tôt au cours du printemps, alors que les semis tardifs ne sont pas encore infestés. Il en résulte des niveaux de populations beaucoup plus élevés sur les premiers que sur les seconds, particulièrement dans le cas de $R$, padi.

Il est, de plus, probable que les semis précoces permettent, au même titre que les repousses, le développement de pucerons ailés qui contaminent les semis tardifs.

L'action des Entomophthorales et des Hyménoptères parasites ne semble pas fortement influencée par la date de semis.

De cette étude, il ressort, d'une part, que les semis de blés d'hiver effectués en octobre sont susceptibles d'être contaminés dès leur levée par des pucerons, donc par la jaunisse nanisante de l'orge, d'autre part que, au moins dans les régions océaniques, s'ajoute au risque précédent, celui de dégâts directs au printemps, plus importants pour les blés d'hiver semés précocement que pour ceux semés plus tardivement au cours de l'automne.

Mots clés additionnels : Anholocyclie, semis précoces, semis tardifs, régions océaniques. on the infestation of fields by Rhopalosiphum padi (L.), Sitobion avenae (F.) and Metopolophium dirhodum (Wlk.) and on the development of aphid populations during the following spring.

In $1977,1978,1979,1980$ and 1982, the development of populations of $S$. avenae, R. padi and $M$. dirhodum was compared on early (October) and late (November) sown fields of wintei wheat. The early sown fields were, in each case, infested before winter by at least 2 species of aphids. The late sown fields were never infested by aphids before the next spring.

When the winter was especially cold (in 1978-1979), the virginopareae overwintering on the early sown fields were destroyed and both types of fields were infested at the same time during the spring. The aphid populations then reached the same size or were a little larger on the late sown fields, probably because of phenological differences $(1979)$.

In all the other cases $(1978,1980,1982)$, virginopareae of the 3 species overwintered on the early sown fields and their populations increased very early in the spring when the late sown fields were not yet infested. The results showed much higher levels of populations on the early sown fields than in the late sown ones, especially concerning $R$. padi.

The study of the structure of aphid populations on early sown fields showed that these fields could be a local reservoir of alates for the contamination of the late sown ones, as the volunteers are.

The role of Entomophthorales and parasitoids did not seem strongly influenced by the sowing date. 
This study shows two risks : 1) early sown (October) winter wheat fields may be infested at or after emergence by aphids and consequently by B.Y.D.V. ; 2) at least in oceanic areas aphids may additionally cause direct damage in spring, more severe for early sown than for late sown winter wheat.

Additional key words : Anholocycly, early sowings, late sowings, oceanic areas.

\section{INTRODUCTION}

Sitobion avenae (F.), Metopolophium dirhodum (Wlk.) et Rhopalosiphum padi (L.), qui sont les principales espèces de pucerons colonisant les céréales à pailles, apparaissent assez polyphages au sein de la famille des Graminées (RAUTAPÄÄ, 1970). Elles peuvent ainsi se reproduire parthénogénétiquement pendant une grande partie de l'année sur différentes cultures (céréales d'hiver, de printemps, maïs, graminées fourragères) dont les périodes de végétation se succèdent dans le temps (DEDRYVER et al., 1983), la production cyclique de formes ailées leur permettant de passer de l'une à l'autre au cours des différents vols d'émigration, dissémination et rémigration (ou retour) (ROBERT \& ROUZE-JOUAN, 1976).

Les populations de pucerons ailés produites au cours de l'automne sur le maïs, les repousses de céréales et les graminées fourragères, peuvent être constituées de plusieurs types d'individus :

D'une part, des mâles et des gynopares qui effectuent leur vol de rémigration sur les hôtes primaires (dans le cas de $R$. padi et de $M$. dirhodum), ou qui colonisent d'autres graminées que celles sur lesquelles ils ont été produits (dans le cas de $S$. avenae) : cette fraction de la population, ou bien est sexuée (mâles), ou bien engendrera des sexués (gynopares) qui pondront des œufs d'hiver diapausants.

D'autre part, des virginipares ailés pouvant constituer l'essentiel des populations ailées, voire la presque totalité dans les régions océaniques, qui contaminent au cours de leur "vol d'automne" d'autres graminées et s'y reproduisent parthénogénétiquement pendant un temps plus ou moins long, selon les aléas du climat hivernal.

C'est la transmission par les pucerons appartenant à cette $2^{\mathrm{e}}$ catégorie (essentiellement par $R$. padi) d'une grave maladie à virus des céréales, la jaunisse nanisante de l'orge (J.N.O.), qui a attiré l'attention des praticiens sur le fait que les jeunes semis de céréales d'hiver pouvaient, dès l'automne, être infestés par ces pucerons (et donc contaminés par la J.N.O.) pour peu que les vols se prolongent après leur période de levée. On a ainsi montré l'importance de la date de semis de ces céréales sur l'épidémiologie de la J.N.O. et la possibilité de limiter les contaminations en évitant les semis précoces ou en les traitant chimiquement à bon escient (LOWE, 1967 ; BAYON et al., 1980b).

Nous avons également montré (DEDRYVER \& GELLÉ, 1982) qu'assez systématiquement dans les régions océaniques et au moins occasionnellement ailleurs, les pucerons des céréales peuvent hiverner à l'état de populations anholocycliques sur des graminées occupant le sol pendant tout l'hiver, telles que les repousses de blé ou d'orge, et que leurs populations pouvaient s'y développer très tôt à la fin de cette sai- son, à la faveur des premiers réchauffements. Les céréales d'hiver semées précocement en automne sont, elles aussi, contaminées avant l'hiver : il était important de déterminer si, comme les repousses, elles étaient susceptibles d'héberger des populations anholocycliques au cours de cette saison, et surtout quelles en étaient les conséquences sur le développement ultérieur des populations. Dans ce but, nous avons comparé pendant 5 années consécutives, dans le Bassin de Rennes, les modalités de contamination par les pucerons puis d'infestation par ceux-ci, de parcelles de blé d'hiver semées pour les premières au cours de la $1^{\text {re }}$ quinzaine d'octobre, pour les secondes, au cours de la $1^{\text {re }}$ quinzaine de novembre.

\section{MATÉRIEL ET MÉTHODES}

Les expérimentations ont été réalisées d'octobre 1977 à juin 1982 sur le domaine du Centre de Recherches I.N.R.A. de Rennes, au Rheu (35), dans des parcelles de blé d'hiver, de variété "Champlein ", semées à 2 dates différentes, en octobre et en novembre de chaque année, comme l'indique le tableau 1. Une exception concerne la parcelle semée en novembre 1980, qui a été emblavée avec le cultivar « Arminda ». Dans le texte qui suit, l'appellation " semis précoces " se rapporte aux parcelles de blé semées en octobre alors que les "semis tardifs" concernent les parcelles emblavées en novembre.

TABLEAU 1

Dates de semis des parcelles d'expérimentation de 1977 à 1981. Dates of sowing of the experimental plots from 1977 to 1981.

\begin{tabular}{lrrrrr}
\hline \hline & 1977 & 1978 & 1979 & 1980 & 1981 \\
\hline Semis « précoce 》 & 8 oct. & 17 oct. 16 oct. 15 oct. 14 oct. \\
Semis « tardif 》 & 8 nov. & 16 nov. 19 nov. 12 nov. 17 nov. \\
\hline \hline
\end{tabular}

En 1977-1978 et 1979-1980, le dispositif était constitué, pour chaque date de semis, de 3 (1977-1978) ou 2 (1979-1980) bandes parallèles alternées, mesurant chacune $10 \times 100 \mathrm{~m}$. En $1978-1979$ et $1981-1982$ pour chaque date de semis, et en 1980-1981 pour le semis précoce seulement, il a été utilisé des parcelles de $7 \mathrm{~m}^{2}$ dans un dispositif bloc à 4 répétitions.

L'estimation des populations de pucerons est faite à partir de prélèvements de talles effectués dans les parcelles et ramenés au laboratoire pour dépouillement. Selon les années et les situations, un nombre variable de talles consécutives est prélevé dans des placettes régulièrement réparties dans les parcelles. Le total pré- 
levé étant toujours compris entre 50 et 300 talles par date de semis et par prélèvement.

Les comptages et déterminations de pucerons vivants, mycosés, et de momies de parasites sont effectués à la loupe binoculaire (DEDRYVER, 1978).

La courbe de vol de $R$. padi, $S$. avenae et $M$. dirhodum, à l'automne 1977, a été obtenue à partir de captures au piège à eau coloré en jaune « Ripolin 514 », au Rheu. Les courbes suivantes (1978, 1979, 1980 et 1981) correspondent aux captures effectuées au piège à succion de 12,2 $\mathrm{m}$ de hauteur, du Rheu.

Les données climatiques sont celles du poste météorologique I.N.R.A. du Rheu. Les stades phénologiques du blé sont repérés selon l'échelle de ZADOKS (ZADOKS et al., 1977). L'échelle de temps est comptée en semaines normalisées (LEWIS \& TAYLOR, 1967).

\section{RÉSULTATS}

\section{A. Infestation des semis à l'automne}

La figure 1 situe les dates de levée des parcelles de blé par rapport aux périodes de capture des 3 principales espèces de pucerons des céréales au piège jaune (en 1977) ou au piège à succion (de 1978 à 1981), au cours des 12 dernières semaines de l'année.

Les périodes de capture d'ailés de $R$. padi se prolongent dans tous les cas pendant plusieurs semaines après la levée des semis précoces (3 semaines en 1977,

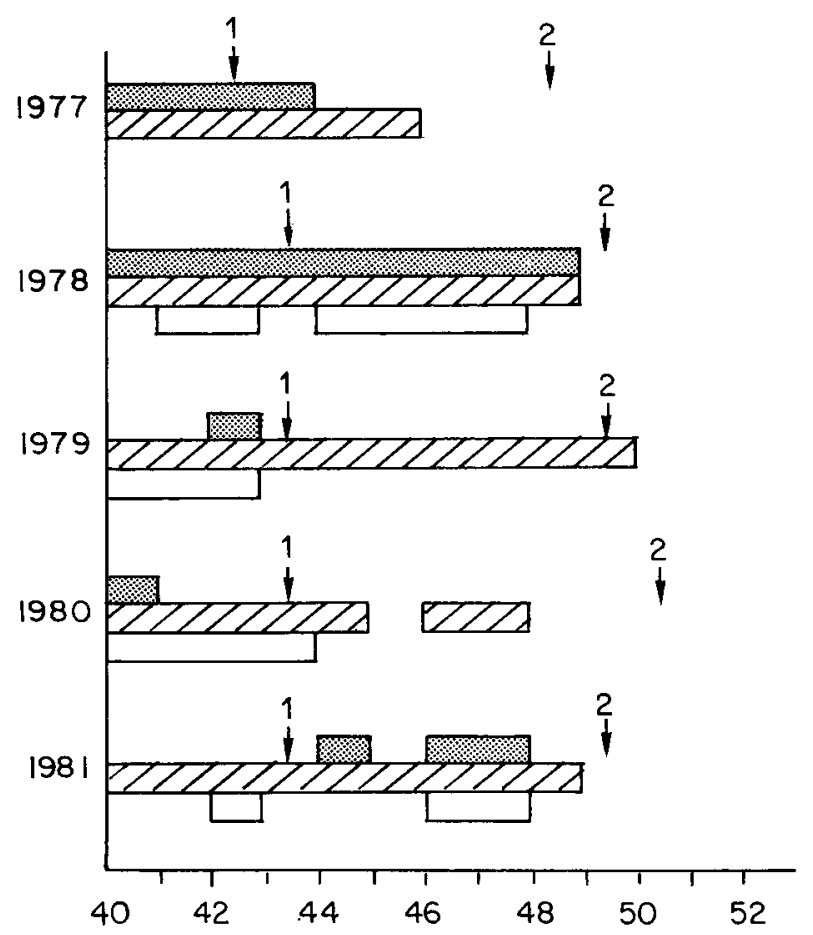

Figure 1

Localisation de la date de levée du blé d'hiver. $(1$ : semis précoce, 2 : semis tardif) par rapport aux périodes de captures de $\mathrm{S}$. avenae 2 , R. padi $\mathrm{ZTh}$ et $\mathrm{M}$. dirhodum $\square$ au piège jaune (en 1977) et au piège à succion (de 1978 à 1981) au cours des 12 dernières semaines de l'année.

Localization of the date of emergence of winter wheat. (1: early sowing; 2 : late sowing) in relation to the periods of capture of $\mathrm{S}$. avenae $\mathrm{R}$. padi $\mathrm{VD}$ and $\mathrm{M}$. dirhodum. $\square$ in yellow trays (1977) and in suction trap (1978-1981) during the last 12 weeks of the year.
5 en 1978, 6 en 1979, 4 en 1980 et 5 en 1981). Elles sont terminées dans 4 cas sur 5 avant celle des semis tardifs. En 1979, les captures s'arrêtent la semaine correspondant à la levée de ces derniers.

Comparativement à celles de $R$. padi, les captures automnales de $S$. avenae et de $M$. dirhodum sont faibles (ce qui n'est pas indiqué par la figure 1), sporadiques et en général beaucoup plus limitées dans le temps. Elles peuvent même être nulles dans le cás de $M$. dirhodum en 1977. Les semis tardifs lèvent toujours au moins une semaine après la fin des captures. Selon les années, les semis précoces lèvent avant la fin des captures de $S$. avenae (en 1977, 1978 et 1981) et de M. dirhodum (en 1978, 1980 et 1981) ou après celle-ci (en 1979 et 1980 dans le cas de $S$. avenae, en 1979 dans celui de $M$. dirhodum). En 1977, aucun M. dirhodum n'est capturé au piège jaune, au cours de l'automne.

\section{TABLEAU 2}

Infestation des semis précoces de blé par les pucerons au cours de l'automne, en nombre de pucerons pour 20 talles. * en novembre 1979 on a simplement noté la présence $(+)$ ou l'absence (-) des espèces considérées, sans en apprécier la quantité. Infestation of early sown plots by aphids during autumn (number of aphids $\mathbf{2 0}$ tillers).

* in November 1979 presence (+) or absence (-) of the 3 species was only checked, without any estimation of their abundance.

\begin{tabular}{lccccc}
\hline \hline & $\begin{array}{c}1977 \\
\text { (26 oct. })\end{array}$ & $\begin{array}{c}1978 \\
(29 \text { nov. })\end{array}$ & $\begin{array}{c}* 1979 \\
(20 \text { nov. })\end{array}$ & $\begin{array}{c}1980 \\
(15 \text { nov. })\end{array}$ & $\begin{array}{c}1981 \\
\text { (5 déc.) }\end{array}$ \\
\hline R. padi & 1,7 & 3 & + & 0,4 & 3,6 \\
S. avenae & 1,2 & 7 & + & 0,05 & 0,1 \\
$M$. dirhodum & 0,3 & 0 & + & 0 & 0 \\
\hline
\end{tabular}

Les résultats indiqués au tableau 2 complètent l'analyse précédente : les observations effectuées une seule fois chaque année au cours de l'automne montrent que les semis précoces sont dans tous les cas contaminés avant l'hiver par $R$. padi et par $S$. avenae bien que, dans 2 cas sur 5 , les captures de ce dernier puceron aient cessé avant la levée des plantules. A l'opposé, les semis précoces semblent beaucoup plus irrégulièrement contaminés par $M$. dirhodum ( 2 cas sur 5) et ce, les années où on aurait pu penser a priori qu'ils ne devaient pas l'être : la $1^{\text {re }}$ fois en 1977, alors qu'aucun vol n'est décelé, et la $2^{\mathbf{e}}$ en 1979 , alors que les captures s'arrêtent une semaine avant la date de levée du blé. Par contre, les semis précoces restent indemnes de $M$. dirhodum durant les automnes 1978 , 1980 et 1981, au cours desquels les vols de ce puceron se sont pourtant prolongés après la date de levée des plantules.

Des sondages similaires ont été effectués fin décembre sur les semis tardifs et ont confirmé que ceux-ci étaient indemnes de pucerons à l'entrée de l'hiver.

\section{B. Etude comparative du développement des popula- tions de pucerons au printemps en fonction de la date de semis}

Une brève analyse de quelques composantes du climat des 5 hivers qui nous intéressent est fournie par le 
TABLEAU 3

Quelques paramètres climatiques permettant de caractériser l'automne et l'hiver, de 1977-1978 à 1981-1982 dans le Bassin de Rennes. (1) température moyenne mensuelle,

(2) minimum mensuel le plus bas (s'il est inférieur à 0 ),

(3) nombre de jours de gel.

Some climatic parameters characterizing autumn and winter from 1977-1978 to 1981-1982 in the area of Rennes.

(1) mean monthly temperatures,

(2) absolute monthly minimum (if below zero),

(3) number of frost days.

\begin{tabular}{|c|c|c|c|c|c|c|c|c|c|c|c|c|c|c|c|}
\hline & \multicolumn{3}{|c|}{$1977-1978$} & \multicolumn{3}{|c|}{$1978-1979$} & \multicolumn{3}{|c|}{$1979-1980$} & \multicolumn{3}{|c|}{$1980-1981$} & \multicolumn{3}{|c|}{$1981-1982$} \\
\hline & 1 & 2 & 3 & 1 & 2 & 3 & 1 & 2 & 3 & 1 & 2 & 3 & 1 & 2 & 3 \\
\hline Octobre & 13 & - & - & 12,1 & $-0,2$ & 1 & 12,4 & - & - & 7,8 & $-0,2$ & 1 & 11,5 & - & - \\
\hline Novembre & 8,1 & $-3,9$ & 5 & 8,3 & -3 & 8 & 7,4 & -3 & 4 & 6,2 & $-3,8$ & 10 & 9,1 & $-1,7$ & 4 \\
\hline Décembre & 6,8 & $-2,7$ & 8 & 6,1 & $-4,5$ & 9 & 7,1 & -2 & 6 & 5,3 & $-4,6$ & 10 & 5,3 & $-3,1$ & 5 \\
\hline Janvier & 4,9 & $-2,5$ & 9 & 1,1 & -7 & 24 & 2,7 & $-6,8$ & 16 & 6 & $-3,5$ & 5 & 6,4 & $-4,5$ & 7 \\
\hline Février & 5,5 & $-7,9$ & 8 & 5,2 & $-7,7$ & 6 & 7,3 & $-0,5$ & 2 & 4 & -5 & 14 & 6,6 & $-1,9$ & 2 \\
\hline Mars & 8,3 & $-1,8$ & 3 & 6,9 & -3 & 8 & 6,2 & $-4,5$ & 5 & 10,1 & $-0,7$ & 1 & 7,3 & -2 & 3 \\
\hline Total (Nj gel) & & & 33 & & & 56 & & & 33 & & & 41 & & & 21 \\
\hline
\end{tabular}

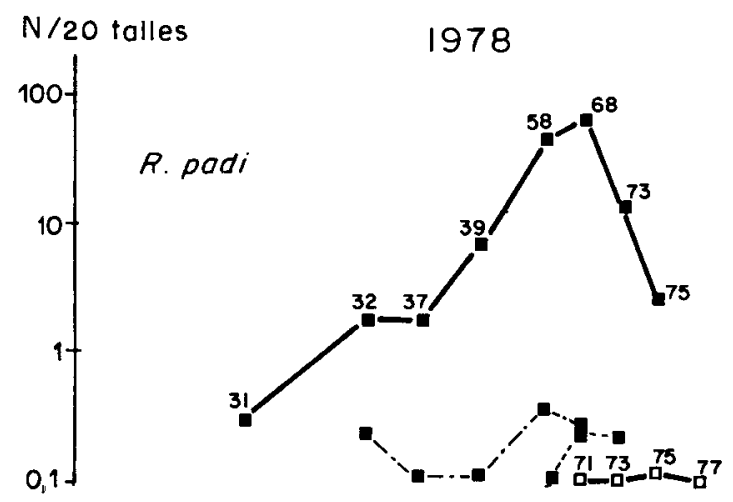

S. avenoe
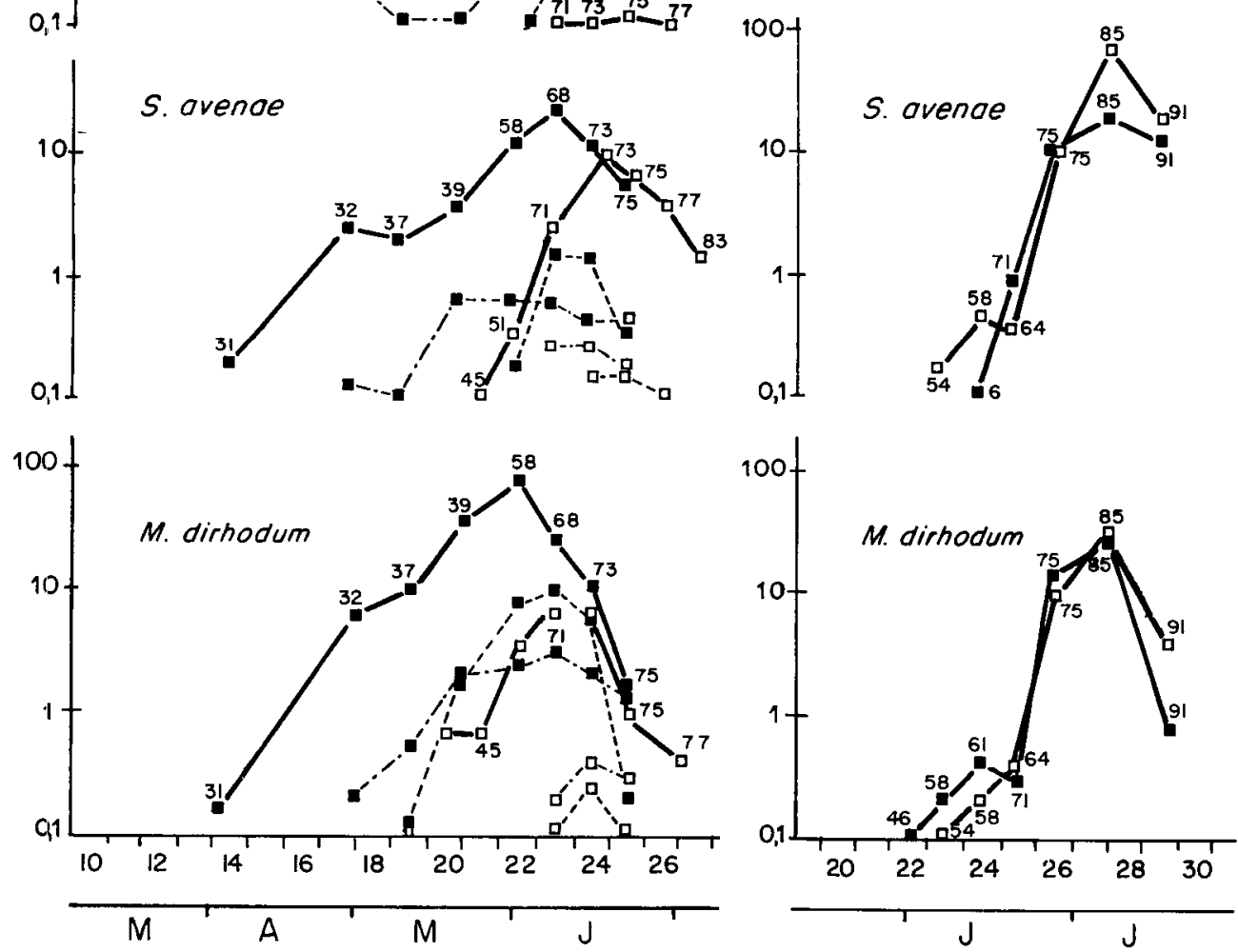

Figure 2

Populations de pucerons des céréales, de pucerons mycosés et de momies en fonction du temps (en abscisse : numéro de la semaine conventionnelle) en 1978 et 1979.

- semis précoces, $\square$ semis tardifs, - nombre total de pucerons, -.-- nombre de pucerons mycosés, -.- nombre de momies. Les nombres représentent les stades du blé selon l'échelle de Zadoks.
Populations of cereal aphids, of aphids killed by fungi and of mummies vs. time (week number) in 1978 and 1979.

- early sowing, $\square$ late sowing, - total amount of aphids, ---number of aphids killed by fungi, - - number of mummies. Numbers represent the stages of wheat (decimal scale). 
tableau 3. Les minima les plus bas ne sont jamais inférieurs à $-8{ }^{\circ} \mathrm{C}(-7,9$ en février 1978 , - 7,7 en février 1979, $-6,8$ en janvier 1980 , -5 en février 1981 et $-4,5$ en janvier 1982). Le nombre de jours de gel est extrêmement variable selon les années au cours de la période considérée : de 21 en 1981-1982 à 56 (dont 24 en janvier) en 1978-1979.

En ce qui concerne l'évolution des populations de pucerons, on ne présentera que 4 années de résultats, ceux de l'année 1981 étant biaisés par le fait que les 2 semis n'étaient pas emblavés avec le même cultivar.

\section{Evolution des populations de pucerons en 1979}

Cette année (fig. 2) s'oppose aux 3 autres, quelle que soit l'espèce de puceron : les populations anholocycliques, présentes au cours de l'automne précédent sur le semis précoce, ont été détruites par les froids du mois de janvier, et les parcelles, quelle que soit leur date de semis, sont contaminées à peu près en même temps par $S$. avenae et $M$. dirhodum, entre la $23^{\mathrm{e}}$ et la $24^{\mathrm{e}}$ semaine conventionnelle, soit entre le 4 et le 17 juin. Etant contaminés en même temps, les 2 semis supportent ensuite des populations qui évoluent sans décalage marqué les unes par rapport aux autres. Dans le cas de $M$. dirhodum, les maxima atteints sont exactement les mêmes pour les 2 dates de semis. Pour $S$. avenae, le maximum atteint est 3 fois plus faible sur le semis précoce que sur le semis tardif ; $R$. padi est pratiquement absent.

2. Evolution des populations de pucerons en 1978, 1980 et 1982

Au cours de ces années (fig. 2 et 3), l'évolution printanière des populations de pucerons est très différente selon la date de semis.
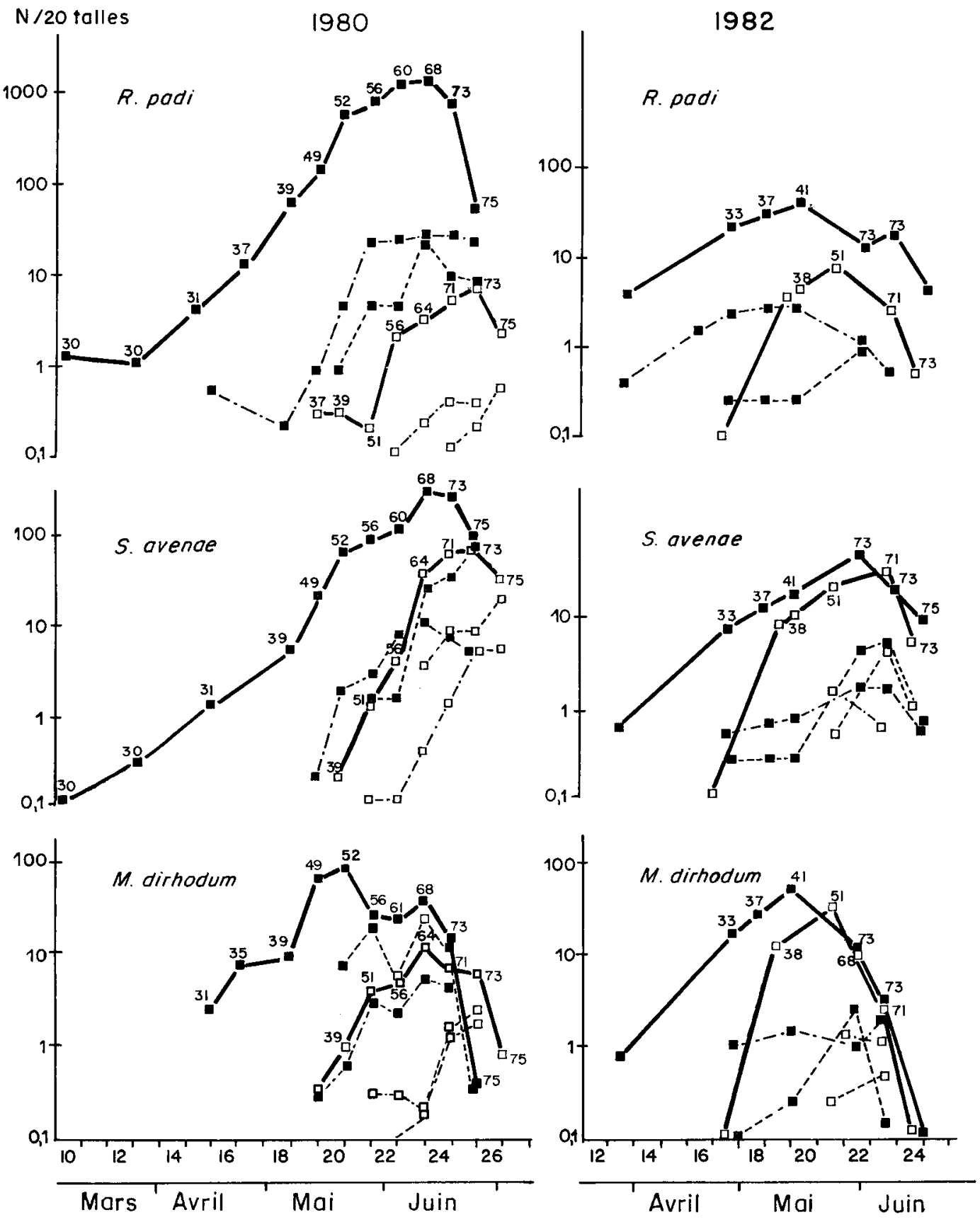

Figure 3

Même légende que la figure 2 pour 1980 et 1982.

Same legends as figure 2 for 1980 and 1982. 


\section{a) Modalités de contamination des parcelles}

En 1978, les $1^{\text {ers }}$ prélèvements sont effectués pendant la $14^{\mathrm{e}}$ semaine conventionnelle (le 6 avril). Le semis précoce héberge de faibles populations des 3 espèces de pucerons dès cette date. Le semis tardif n'est contaminé qu'au cours des $19^{e}$ semaine (10 mai) par $M$. dirhodum, $21^{\mathrm{e}}$ semaine (24 mai) par $S$. avenae et $23^{\mathrm{e}}$ semaine ( 8 juin) par $R$. padi.

En 1980 , les $1^{\text {ers }}$ prélèvements sont faits durant la $10^{\mathrm{e}}$ semaine (6 mars). A cette date, le semis précoce héberge déjà des populations non négligeables de $R$. padi (1 pour 20 talles) et quelques $S$. avenae $(0,1$ pour 20 talles); il paraît par contre indemne de $M$. dirhodum qu'on ne trouvera qu'à la $15^{\mathrm{e}}$ semaine (15 avril). Le semis tardif est contaminé la 19e semaine (13 mai) par $R$. padi et $M$. dirhodum et au cours de la $20^{\mathrm{e}}$ semaine (19 mai) par $S$. avenae.

En 1982 , les $1^{\text {ers }}$ prélèvements sont effectués pendant la $13^{\mathrm{e}}$ semaine conventionnelle $(26$ mars) et le semis précoce est, à cette date, contaminé par les 3 espèces. Le semis tardif n'est contaminé qu'au cours de la $17^{\text {e }}$ semaine ( 27 avril) par les 3 espèces.

b) Evolution ultérieure des populations de pucerons (fig. 2 et 3 )

- $R$. padi : c'est pour cette espèce que les différences de populations entre dates de semis sont les plus marquées : Les maxima notés, pour 20 talles, sont respectivement, pour le semis précoce et le semis tardif, de 72 et 0,3 en 1978,1400 et 7 en 1980,40 et 7 en 1982, année où les différences sont moins fortes.

Dans le cas des semis précoces, les maxima des populations sont atteints au stade 68 (pleine floraison) en 1978 et 1980, et au stade 41 (gonflement) en 1982. Ils interviennent à des stades plus avancés sur les semis tardifs : 73-75, soit début à mi-laiteux en 1978 et 1980, 51, soit début épiaison en 1982.

- $S$. avenae: en 1978, les populations atteignent un maximum de 25 pucerons pour 20 talles sur semis précoces, soit plus du double du niveau obtenu sur le semis tardif (10/20 talles). En 1980, le maximum est de 300 pucerons pour 20 talles sur semis précoce contre 65 sur semis tardif. Enfin en 1982, les différences sont moins importantes, le maximum est de 45 pucerons pour 20 talles sur le semis précoce au lieu de 32 sur le semis tardif.

- M. dirhodum: Les populations observées pour 20 talles s'élèvent, respectivement pour le semis précoce et le semis tardif, à 80 et 7 en 1978,100 et 13 en 1980,50 et 30 en 1982 . c) Les semis précoces, producteurs d'ailés de contamination au printemps

Le tableau 4 indique qu'il y a une bonne coincidence entre le début de la formation des pucerons ailés sur les semis précoces (estimée par anticipation en évaluant la production de larves à ptérothèques) et la période de contamination des semis tardifs par les mêmes espèces. Il y a ainsi de fortes présomptions pour que les semis tardifs aient été contaminés au printemps par des ailés issus de semis précoces (ceux faisant l'objet de notre étude, ou d'autres à peu près similaires et situés dans les environs).

\section{Influence de la date de semis sur le rôle des Hyménoptères parasites et celui des mycoses à Entomophthorales}

\section{Premières observations (fig. 2 et 3 )}

Des momies, appartenant aux 3 espèces de pucerons, sont observées sur le semis précoce dès la fin avril (semaine 17) en 1978. En 1980, on trouve des momies de $R$. padi dès la mi-avril (semaine 15 ) et des momies de $S$. avenae et $M$. dirhodum dès le début mai (semaine 19). Enfin, en 1982, des momies de $R$. padi se rencontrent dès la fin mars (semaine 13) et celles de $S$. avenae et de $M$. dirhodum seulement à la fin avril (semaine 17).

Dans les semis précoces, pour peu que ceux-ci soient encore contaminés au début du printemps, il semble donc que l'activité des Hyménoptères parasites commence très tôt spécialement dans les foyers de $R$. padi. Par contre, on y observe systématiquement les premiers pucerons mycosés plus tard que les premières momies : entre la $19^{\mathrm{e}}$ et la $22^{\mathrm{e}}$ semaine en 1978 , entre la $20^{\mathrm{e}}$ et la $21^{\mathrm{e}}$ en 1980 , entre la $17^{\mathrm{e}}$ et la $18^{\mathrm{e}}$ en 1982.

Dans les semis tardifs, momies et mycosés sont généralement trouvés très tardivement, nettement après la contamination des parcelles par les pucerons.

\section{Effet des Entomophthorales et des Hyménoptères parasites sur les populations de pucerons des différentes dates de semis}

Ces 2 types d'ennemis naturels se manifestent dans presque tous les cas. Les exceptions concernent l'année 1979, où leur action est pratiquement nulle quelle que soit la date de semis, et l'année 1982, au cours de laquelle on ne récolte ni momie ni puceron

TABLEAU 4

Relation entre les périodes de début de production de larves à ptérothèques des pucerons des céréales dans les semis précoces de blé (l) et les dates de contamination approximatives des semis tardifs (2). Les numéros de semaines conventionnelles sont entre parenthèses.

Relation between the start of the winged cereal aphid nymph production infestation in the early sown plots of wheat (1) and the rough dates of contamination of the late sown plots (2). The numbers of the weeks between brackets.

\begin{tabular}{|c|c|c|c|c|c|c|}
\hline & & 1978 & 1979 & 1980 & 1981 & 1982 \\
\hline \multirow{2}{*}{ S. avenae } & $\int 1$ & $28 / 4(17)$ & $20 / 6(25)$ & $24 / 4(17)$ & $7 / 4(14)$ & $26 / 3(13)$ \\
\hline & 2 & $26 / 4(17)$ & $6 / 6(23)$ & $13 / 5(19)$ & $14 / 4(15)$ & $15 / 4(15)$ \\
\hline \multirow{2}{*}{ M. dirhodum } & $\int 1$ & $28 / 4(17)$ & $20 / 6(25)$ & $24 / 4(17)$ & $7 / 4(14)$ & $26 / 3(13)$ \\
\hline & $\{2$ & $26 / 4$ (17) & $13 / 6(24)$ & $13 / 5$ (19) & $14 / 4(15)$ & $15 / 4(15)$ \\
\hline \multirow{2}{*}{ R. padi } & $\int 1$ & $19 / 5(20)$ & - & $25 / 3$ (12) & $7 / 4(14)$ & $26 / 3$ (13) \\
\hline & $\{2$ & $24 / 5$ (21) & - & $13 / 5$ (19) & $14 / 4(15)$ & $2 / 4$ (14) \\
\hline
\end{tabular}




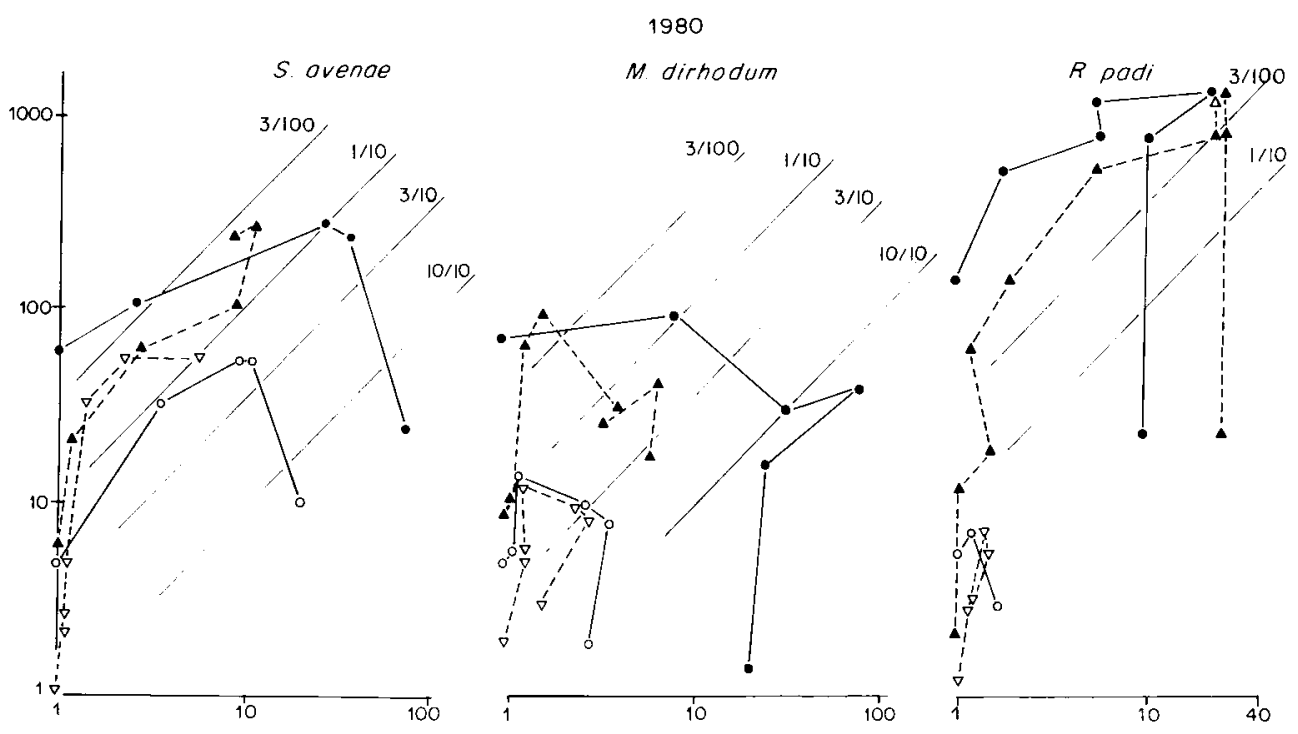

Figure 4

Courbes d'évolution simultanée du nombre de pucerons vivants (en ordonnée) et du nombre de pucerons mycosés (en abscisse) sur les semis précoces (- - ) et tardifs (- $\bigcirc)$.

Courbes d'évolution simultanée du nombre de pucerons vivants (en ordonnée) et du nombre de momies (en abscisse) sur les semis précoces (-- $\mathbf{\Lambda}--)$ et tardifs (--- $\nabla---)$.

* les résultats sont exprimés en $N+1$ pucerons/20 talles.

mycosé dans les populations de $R$. padi des semis tardifs.

A titre d'exemple, on analysera la situation de l'année 1980 (fig. 4). Quelle que soit l'espèce de puceron, une grande similitude s'observe dans le tracé des courbes d'évolution simultanée pucerons vivants/pucerons mycosés, d'une part, et pucerons vivants/momies, d'autre part, en fonction de la date de semis : les 2 courbes se rapportant à chacun des ennemis naturels sont grossièrement homothétiques l'une de l'autre d'un facteur correspondant au rapport des nombres de pucerons présents respectivement sur semis précoce et sur semis tardif. L'action des Hyménoptères parasites et des Entomophthorales ne semble donc pas être fortement influencée par la date de semis. Ainsi, chez $S$. avenae, pour chaque date de semis, les rapports momies/vivants, d'une part, et mycosés/vivants, d'autre part, évoluent au cours du temps dans les mêmes limites : entre $3 / 100$ et $1 / 10$ pour les $1^{\text {ers }}$ et entre $3 / 100$ et $20 / 10$ pour les $2^{\text {es }}$. Quelle que soit la date de semis, les Hyménoptères parasites exercent donc au cours du temps une pression parasitaire assez faible et constante, alors que l'action des Entomophthorales évolue en épizootie. Tandis qu'un processus identique s'observe chez $M$. dirhodum, dans le cas de R. padi, malgré les très fortes populations de ce puceron, l'action des Entomophthorales est beaucoup plus faible que sur les 2 autres espèces et se montre même inférieure à celle des Hyménoptères parasites.

\section{DISCUSSION ET CONCLUSIONS}

Les résultats que nous avons obtenus au cours de ces expérimentations nous permettent de tirer 2 catégories de conclusions concernant des situations écogéographiques différentes.

A. D'une part, ils confirment que, dans le Bassin de Rennes, les semis de céréales d'hiver effectués dans
Co-development of the numbers of living aphids (ordinate) and aphids killed by fungi (abscissa) on early (- $\rightarrow$ and late sowings (-O-).

Co-development of the numbers of living aphids (ordinate) and mummies (abscissa) on early (--- $\Delta---)$ and late sowings ( $--\nabla-\cdots)$.

* results expressed as $\log (N+I)$ aphids $/ 20$ tillers.

la $1^{\text {re }}$ quinzaine d'octobre ont de fortes chances d'être contaminés avant l'hiver par 2 ou 3 espèces de pucerons des céréales dont une ( $R$. padi) est un excellent vecteur d'une souche grave de la jaunisse nanisante de l'orge (LAPIERRE, 1976). La grave épidémie de J.N.O., observée dans tout l'Ouest de la France en 1982 , en est une récente illustration et les facteurs gouvernant l'épidémiologie de la J.N.O. en fonction des conditions culturales, du climat, des réservoirs de pucerons, etc... ont été étudiés de façon approfondie par BAYON (BAYON $e t a l ., 1980 a$ et $b$; BAYON et $a l$, 1982).

L'analyse des captures de pucerons des céréales obtenues par le réseau de pièges à succion mis en service en France depuis 1978 (MOUCHART, 1981) permet de généraliser ce type de conclusion à la plus grande partie des régions céréalières françaises puisque, presque partout, les vols de $R$. padi se prolongent au moins jusqu'aux derniers jours de novembre (Evelyne TURPEAU, comm. pers.). On n'a évidemment pas l'assurance que tous les vols sont fidèlement enregistrés par le piège à succion et, en particulier, que des vols courts d'une parcelle à l'autre ne se prolongent pas après la fin des captures. Cette éventualité expliquerait pourquoi, dans nos expérimentations, les semis précoces ont toujours été contaminés par S. avenae, alors qu'en 1979 et 1980 il n'y avait pas coïncidence entre la période de capture des ailés et la levée des plantules. De même, il semble que dans l'Ouest, en 1981-1982, des semis tardifs aient pu aussi être contaminés avant l'hiver par $R$. padi, ce qui indique que quelques individus ont probablement encore pu voler après la fin des captures.

B. Il apparaît d'autre part que, dans les régions océaniques où les hivers "doux " permettent assez généralement le maintien de populations anholocycliques de pucerons sur graminées au cours de la mauvaise saison, les céréales contaminées avant l'hiver par 
les pucerons peuvent comme les repousses (DEDRYVER \& GELLÉ, 1982), le rester au cours de celui-ci. Dans ce cas, les colonies aphidiennes déjà sur place au tout début du printemps bénéficient d'un avantage considérable qui leur permet de se multiplier activement dès les premiers réchauffements. Elles sont, d'une part, productrices d'ailés pouvant participer à la contamination des semis tardifs et, d'autre part, elles atteignent des niveaux de population bien supérieurs à ce qu'ils sont sur des semis tardifs contaminés beaucoup plus tard au printemps. Une technique culturale peut donc, au moins dans certains cas, influer sur la nuisibilité directe de ces pucerons puisque en 1978, et à plus forte raison en 1980 dans nos expérimentations, il a pu y avoir dégâts sur les semis précoces avec dépassement d'un seuil théorique de 10 pucerons par talle, tandis que les semis tardifs demeuraient indemnes. Le fait que les différences les plus fortes soient observées pour $R$. padi correspond parfaitement au fait que, dans les zones océaniques, les seules pullulations graves de ce puceron affectent, dans pratiquement tous les cas, des céréales semées en début d'automne, alors que les populations de $R$. padi, soit ne s'établissent pas, soit se développent très peu sur les céréales qu'elles contaminent au cours du printemps.

La différence de "réponse » des 3 espèces de pucerons au facteur "date de semis " semble liée au fait que, dans le Bassin de Rennes, les différences phénologiques entre parcelles semées en octobre et en novembre sont très importantes jusqu'à la fin de la $1^{\text {re }}$ quinzaine de mai, c'est-à-dire au début d'épiaison des semis précoces, puis s'atténuent ensuite très rapidement pour devenir faibles au stade floraison. Sur les semis précoces, la montaison commence tôt et dure longtemps du fait des températures encore fraîches ( 9 semaines en 1980, au moins 7 en 1978, 6 en 1979, 7 en 1982) ; il semble que ce stade phénologique soit le plus favorable au développement de $R$. padi qui forme des ailés en abondance dès la floraison (ADAMS \& DREW, 1964), alors qu'à l'inverse, une contamination des cultures à un stade phénologique avancé (après la floraison) ne permet pas toujours le développement des populations, comme cela a été le cas en 1978. On peut également avancer l'hypothèse que $R$. padi est mieux établi, à la fin de l'hiver, sur les semis précoces que les 2 autres espèces, et qu'il est peut-être moins sujet qu'elles au parasitisme.

Le rôle, a posteriori, de la date de semis des céréales d'hiver sur le développement au printemps suivant des populations de pucerons qui s'y trouvent doit être compris comme un schéma propre aux régions océaniques. En effet, dans les régions céréalières du Bassin parisien, du Laonnais, de Champagne et du Centre de la France, l'avancée, pour des raisons agronomiques, des dates de semis des céréales d'hiver est bien plus fréquente que dans l'Ouest, mais les froids plus rigoureux (et peut-être la généralisation des traitements aphicides d'automne contre la J.N.O. ...) rendent la survie des populations anholocycliques beaucoup plus problématique au cours de l'hiver. Il n'est donc pas exclu qu'un phénomène inverse de celui que nous avons décrit puisse s'y produire au moins certaines années. Ce phénomène s'apparenterait à ce que nous avons observé dans le Bassin de Rennes en 1979 : la rigueur de l'hiver 1978-1979 y a éradiqué les populations anholocycliques locales persistant sur graminées (DEDRYVER \& GELLÉ, 1982) et les recontaminations de printemps ont été le fait d'apports migratoires ultérieurs, par des pucerons venus d'autres régions et contaminant assez tardivement toutes les céréales en même temps, quel que soit leur passé cultural. Les semis tardifs, qui étaient phénologiquement moins avancés, ont été dans ce cas, autant ou plus infestés que les semis précoces, physiologiquement plus âgés, donc plus "résistants ». Ce processus semble être exceptionnel dans l'Ouest de la France où les hivers sont rarement rudes, mais pourrait être plus fréquent dans des régions plus continentales.

Isolé par l'expérimentation dans la présente étude, l'« effet de la date de semis », qui vient d'être caractérisé, doit aussi être considéré dans le cadre de la pratique agricole où il peut interférer avec d'éventuels effets variétaux puisque les cultivars semés précocement en automne ne sont en général pas les mêmes que les variétés plus ou moins alternatives semées en fin novembre ou décembre. Pour une date de semis donnée, il y a des différences parfois importantes entre les populations de pucerons se développant sur différentes variétés (DEDRYVER et al., 1983) et l'expression de cet effet variétal peut aussi être considérablement modifiée en fonction des dates de semis.

Recu le 30 novembre 1983. Accepté le 5 avril 1984.

\section{REMERCIEMENTS}

Les auteurs remercient vivement Madame Joëlle RouzE-JoUAN (I.N.R.A.-Zoologie, Le Rheu) et Mademoiselle Evelyne TURPEAU (I.T.P.T., St Rémy l'Honoré) qui nous ont permis d'utiliser les résultats de capture au piège jaune et au piège à succion du Rheu.

\section{RÉFÉRENCES BIBLIOGRAPHIQUES}

Adams J. B., Drew M. E., 1964. Grain aphid in New-Brunswick : I. Field development on oats. Can. J. Zool., 42, 735-740.

Bayon F., Ayrault J. P., Pichon P., 1980 a. La Jaunisse Nanisante de l'Orge. Symptômes, dégâts, cycles, moyens de lutte, améliorations. Déf. Vég., 204, 181-202.

Bayon F., Ayrault J. P., Pichon P., 1980 b. Comparaison des aphicides dans la lutte contre les pucerons vecteurs de la Jaunisse Nanisante de l'Orge (BYDV ou JNO). Med. Fac. Landbouw. Rijksuniv. Gent, 45, 529-536.

Bayon F., Ayrault J. P., Pichon P., 1982. Epidémiologie de la Jaunisse Nanisante de l'Orge (BYDV) en Poitou-Charentes. Med. Fac. Landbouw. Rijksuniv. Gent, 47, 1039-1052.
Dedryver C. A., 1978. Biologie des pucerons des céréales dans l'Ouest de la France. I. Répartition et évolution des populations de Sitobion avenae F., Metopolophium dirhodum Wlk. et Rhopalosiphum padi L., de 1974 à 1977 sur blé d'hiver dans le Bassin de Rennes. Ann. Zool. Ecol. Anim., 10, 483-505.

Dedryver C. A., Gellé A., 1982. Biologie des pucerons des céréales dans l'Ouest de la France. IV. Etude de l'hivernation de populations anholocycliques de Rhopalosiphum padi L., Metopolophium dirhodum Wlk. et Sitobion avenae F. sur repousses de céréales dans trois stations de Bretagne et du Bassin Parisien. Acta Oecol. Oecol. Applic., 3, 321-342. 
Dedryver C. A., Di Pietro J. P., Gellé A., Pierre J. S., Tanguy S. 1983. Quelques aspects du rôle des assolements et des pratiques culturales sur les fluctuations de populations de pucerons des céréales en région océanique. Journées d'études "Rotations céréalières intensives », O.N.I.C.-I.N.R.A.-I.T.C.F., 16-17 mai 1983. O.N.I.C. Edit. (à paraître).

Lapierre H., 1976. Problèmes posés par les virus des céréales en France. Déf. Vég.; 178, 66-75.

Lewis T., Taylor L. R., 1967. Introduction to Experimental Ecology, Academic Press, London and New York, $401 \mathrm{p}$.

Lowe A. D., 1967. Avoid yellow dwarf virus by late sowing. $N .-Z$. Wheat Rev., 10, 59-63.

Mouchart A., 1981. Le réseau expérimental ACTAPHID. Les motifs de sa création et présentation. In Les Pucerons des cultures, pp. 65-68. Journées d'études et d'information A.C.T.A., 2-3-4 mars 1981, Paris.

Rautapää J., 1970. Preference of cereal aphids for various cereal varieties and species of Gramineae Juncaceae and Cyperaceae. Ann. Agric. Fenn., 9, 267-277.

Robert Y., Rouzé-Jouan J., 1976. Activité saisonnière de vol des pucerons (Hom. Aphididae) dans l'Ouest de la France. Résultats de neuf années de piégeage (1967-1975). Ann. Soc. Entomol. Fr. (N.S.), 12, 671-690.

Zadoks J. C., Chang T. T., Konzak C. F., 1977. Un code décimal pour les stades de croissance des céréales. Phytiatr.-Phytopharm., 26, $129-140$. 